\title{
LA RADIACIÓN ULTRAVIOLETA Y LA PIEL DEL DEPORTISTA
}

\author{
José Moncada Jiménez
}

\author{
Recibido 28-I-2003 • Aceptado 10-VI-2003
}

\begin{abstract}
Resumen: En este artículo se presenta una revisión de literatura acerca de la relación entre la radiación solar y las lesiones de la piel. Se sabe que una parte de la población se expone diariamente, y a veces durante muchas horas, a la radiación ultravioleta de los rayos solares. Sin embargo, por desconocimiento o por negligencia, muchas personas sufren de lesiones por la exposición aguda o crónica a los rayos solares. En Costa Rica, las lesiones relacionadas con la exposición al sol son comunes; solamente durante el trienio de 1994 a 1996, el cáncer de piel fue el de mayor incidencia entre las mujeres y el segundo entre los hombres. Se ha llegado a determinar que el riesgo para adquirir cáncer de piel aumenta si se poseen ciertas características fenotípicas, como por ejemplo, piel blanca, cabello color rubio o rojo, y ojos color verde o azul. Aún queda por determinar la posible asociación entre la exposición a los rayos ultravioleta en personas que se exponen diariamente durante largos períodos de tiempo a los rayos solares, como por ejemplo los educadores físicos y los deportistas.
\end{abstract}

Palabras clave: Radiación Solar, Lesión de Piel, Prevención, Deportes, Educador Físico.

\section{Introducción}

Diferentes tipos de actividades de la vida diaria se llevan a cabo al aire libre. Ocupaciones profesionales y técnicas, celebraciones, paseos, deportes, y una amplia gama de eventos, permiten que personas de distintos grupos de edad se expongan a la radiación ultravioleta (UV) del sol. Aunque se sabe que la radiación solar en la piel es necesaria para una buena salud, ya que interviene en la transformación de una forma inactiva a una forma activa de la vitamina $\mathrm{D}$, parece que una importante cantidad de personas no conoce los posibles riesgos de la exposición prolongada y crónica a este tipo de radiación.

De acuerdo con Tortora y Grabowski (1993), la exposición intensa, prolongada y excesiva al sol puede provocar cáncer de piel, sin importar la pigmentación de ésta. Anteriormente se pensaba que únicamente las personas con piel de color muy blanco eran quienes contraían cáncer de piel; sin embargo, parece que aún las personas con pigmentación más oscura también pueden sufrir lesiones cancerosas.

Los tres tipos de cáncer de piel causados por la exposición crónica y excesiva al sol son el epitelioma basocelular, el carcinoma epidermoide, y el melanoma. De estos tres tipos de cáncer, el epitelioma 
basocelular es el más común (75\% de todos los casos); sin embargo, los melanomas parecen ser los más agresivos y potencialmente mortales, especialmente en las mujeres jóvenes (Tortora y Grabowski, 1993). En los Estados Unidos de Norteamérica, por ejemplo, el cáncer de piel es el cáncer diagnosticado más frecuentemente, siendo el melanoma el responsable de la mayor cantidad de muertes (Muglia, Pesce, y McDonald, 1999).

Aunque es bien conocida la influencia positiva de la actividad física en la prevención de muchas enfermedades, incluyendo el cáncer de mama en mujeres y el cáncer de colon en hombres (Bernstein, Henderson, Hanisch, Sullivan-Halley, y Ross, 1994; Coogan, Newcomb, Clapp, Trentham-Dietz, Baron, y Longnecker, 1997; Ford, 1999; Friedenreich y Rohan, 1995; Rockhill, Willett, Hunter, Manson, Hankinson, y Colditz, 1999; Thune, Brenn, Lund, y Gaard, 1997; Verloop, Rookus, van der Kooy, y van Leeuwen, 2000; Wyshak, y Frisch, 2000); hasta el día de hoy, pocos investigadores han dirigido su atención a la posible relación entre la exposición prolongada y crónica a la radiación UV en deportistas competitivos o recreativos (Moehrle, Heinrich, Schmid, y Garbe, 2000) o en educadores físicos o personal involucrado diariamente en el planeamiento, dirección y ejecución de actividades al aire libre. Es por ello que se ha querido presentar en esta revisión de literatura, información relacionada con los factores de riesgo para el desarrollo de lesiones en la piel, y describir la evidencia actual acerca de la relación entre la exposición a la radiación UV y la presencia de cáncer en deportistas o en personas que se exponen a la radiación UV durante mucho tiempo. El propósito implícito de la revisión es el de alertar a los docentes, especialmente a los educadores físicos, a que tomen las medidas de prevención necesarias para evitar las lesiones en la piel que podrían desencadenarse en algún tipo de cáncer. Así mismo, se pretende proporcionar guías para la prevención y detección de lesiones en la piel con el propósito de que sean transmitidas a los estudiantes y otras personas que laboran al aire libre.

\section{Factores de riesgo para el cáncer de piel}

Para Tortora y Grabowski (1993), el pigmento natural de la piel no puede brindar una completa protección contra los rayos UV de la luz solar, por lo que pueden aparecer lesiones en la piel (i.e., queratosis solar), las cuales eventualmente podrían convertirse en lesiones cancerosas. Algunos factores de riesgo para el cáncer de piel son el tipo de piel (etnicidad), la raza, el sexo, la exposición al sol (aguda o crónica), historia familiar, edad (el adulto mayor es más propenso), y estado inmunitario (las personas inmunosuprimidas son más susceptibles) (Jerant, Johnson, Sheridan, y Caffrey, 2000; Tortora y Grabowski, 1993).

Algunos investigadores han encontrado un mayor riesgo de adquirir cáncer de piel (especialmente del tipo no melanoma) en las personas de piel blanca, de cabello rubio o rojo, como las de tipo anglosajona o de origen mediterráneo, a quienes se les dificulta broncearse (i.e., ponerse morenas) y que siempre se queman al sol (i.e., se tornan de color rojo) (Rosso, Joris, y Zanetti, 1999; Tortora y Grabowski, 1993). En estudios realizados con poblaciones del Sur de Europa, se ha encontrado que las personas que no se pueden broncear presentan un riesgo estable para carcinoma de células basales (CCB), mientras que aquellas personas a las que no se les dificulta broncearse, desarrollan cáncer únicamente durante exposiciones prolongadas a la radiación UV (Rosso et al., 1996).

En un estudio de tipo cohorte prospectivo realizado por Van Dam et al. (1999), se investigaron algunas características fenotípicas, la exposición al sol, y el 
riesgo para adquirir CCB. Los investigadores contaron con una muestra de 44591 profesionales de la salud, estadounidenses, masculinos, con edades entre 40 y 75 años, y libres de cáncer al momento de ingreso al estudio. A estos sujetos se les dio un seguimiento de 8 años, y en ese período hubo 3273 casos documentados de CCB. Las asociaciones más relevantes para un elevado riesgo para contraer CCB fueron: a) poseer cabello rojo, b) ojos color verde, avellana, o azul, c) tendencia a quemarse la piel (color rojo, no se podían broncear), y d) descendencia europea.

Por otra parte, se han reportado incidencias de cáncer de piel mayores en personas que viven en altitudes, en poblaciones que viven en zonas donde se reciben muchos días con sol al año, en personas que trabajan al aire libre, y en las que han sufrido tres o más quemaduras solares graves; por lo que se infiere que la exposición prolongada al sol podría ser un factor de riesgo importante para la aparición del cáncer en la piel.

\section{Cáncer de piel en deportistas}

La literatura relacionada al tema de la radiación UV y sus efectos en los deportistas es realmente escasa. Sin embargo, existen publicados algunos estudios epidemiológicos en los que se han reportado los riesgos para adquirir una variada cantidad de tipos de cáncer en poblaciones físicamente activas (e.g., atletas universitarios), o en personas con ocupaciones teóricamente activas (e.g., mensajeros) (Wyshak y Frisch, 2000).

Para Moehrle et al. (2000), la radiación UV o la exposición al sol, es el factor de riesgo más importante para el desarrollo de cáncer de piel, ya sea en forma de melanoma o no melanoma. Bajo esta premisa, Moehrle et al. estudiaron a 6 ciclistas profesionales durante 6 etapas del "Tour de Suisse", con el propósito de cuantificar la exposición a la radiación UV durante la competencia y conocer la magnitud de las posibles lesiones por dicha exposición. Los investigadores encontraron que los niveles de exposición personales diarios durante las etapas de esta vuelta ciclística excedían más de 30 veces los límites de exposición UV recomendados internacionalmente. La hipótesis que queda por estudiar es si efectivamente ese tipo de exposición UV crónica podría ser un factor desencadenante de algún tipo de cáncer, y demostrar que el efecto protector que brinda el ejercicio se reduce en las personas que se exponen diariamente durante largos períodos de tiempo a la radiación UV, como es el caso de los deportistas o de los educadores físicos, quienes por la naturaleza de su profesión laboran a veces hasta $7 \mathrm{u} 8$ horas consecutivas bajo el sol.

En un estudio retrospectivo realizado con atletas femeninas ( $\mathrm{n}=2622)$ y no atletas $(n=2776)$, no se encontraron diferencias estadísticamente significativas en las tasas de prevalencia de melanomas malignos y cáncer de piel entre ambos grupos de universitarias (Frisch, Wyshak, Albright, Albright, y Schiff, 1989). A pesar de ello, los hallazgos si fueron consistentes para otros tipos de cáncer (e.g., sistema digestivo, reproductivo, tiroides, vejiga, pulmón, leucemia, linfoma, mieloma, y enfermedad de Hodgkin) cuando se compararon atletas y no atletas, teniendo las atletas una ventaja en la reducción del riesgo para desarrollar el cáncer (Frisch et al., 1985).

Pukkala, Kaprio, Koskenvuo, Kujala, y Sarna (2000), estudiaron la incidencia de cáncer en atletas finlandeses masculinos. Para ello, se obtuvo una cohorte de 2269 atletas de clase mundial nacidos entre 1920 y 1965, de los cuales se obtuvo la incidencia de cáncer durante los años 1967 a 1995. Pukkala et al. (2000), encontraron una tasa de incidencia de 331 casos de cáncer en este grupo de atletas; mientras que en el ámbito nacional la tasa de incidencia era de 404 casos. Se encontró además un 
menor riesgo en cáncer de pulmón, especialmente entre los atletas de resistencia, y en cáncer de riñón. Los autores sugieren que estas reducciones en el riesgo se podrían relacionar con mejores hábitos alimentarios (e.g., más vegetales, menor grasa) y con una marcada reducción en el vicio del fumado de parte de los atletas comparados con no atletas (Pukkala et al., 2000). Sin embargo, también se encontró un riesgo relativo mayor en la aparición de sarcomas óseos y de tejido blando en los corredores, lo cual podría explicarse por las lesiones ocurridas durante su periodo activo como deportistas.

\section{Implicaciones para la salud}

Del estudio de Pukkala et al. (2000), se podría pensar que los corredores que sufren lesiones durante sus años como deportistas activos, podrían tener mayores posibilidades de desarrollar algún tipo de sarcoma óseo o de tejido blando a largo plazo; por lo que es vital que los atletas traten de maximizar las medidas de prevención (e.g., mejores zapatos, mejores atuendos deportivos), y que si se lesionan traten de completar el programa de rehabilitación para evitar alguna recaída.

En el estudio de Moehrle et al. (2000), se encontró que los ciclistas se exponían diariamente a cantidades excesivas de radiación UV. Sin embargo, se requeriría de un estudio longitudinal en los atletas para determinar si existe asociación entre el grado de exposición a la radiación UV y el desarrollo de algún tipo de cáncer de piel.

Finalmente, se han reportado otros tipos de lesiones en la piel y tumores (especialmente carcinoma hepático) en atletas, derivadas del uso de esteroides anabólicos en fisicoculturistas (Creagh, Rubin, y Evans, 1988; Mayerhausen y Riebel, 1989; Mottram y George, 2000); y otros tipos de lesiones relacionadas con el metabolismo del colágeno, producto de traumas frecuentes y fricción, como en el caso de los surfeadores, boxeadores, bolichistas y futbolistas (Cohen, Eliezri, y Silvers, 1992).

En educadores físicos (deportistas y no deportistas) no existen datos que permitan estimar la posibilidad de desarrollar cáncer de piel, pues no se han llevado a cabo estudios sistemáticos; por lo que por el momento no se sabe, por ejemplo, el número de horas que laboran cuando la radiación UV es más fuerte, el tipo de piel, el color de los ojos, y otras características que han sido descritas en otras poblaciones.

\section{Recomendaciones}

Aunque en la mayoría de los países centroamericanos no se cuenta con una población mayoritaria con características como las que describen los autores (i.e., piel blanca, cabello rubio, ojos color verde o azul) (Tortora y Grabowski, 1993; Rosso, Joris, y Zanetti, 1999; Van Dam et al., 1999), es preocupante saber que por ejemplo, en Costa Rica, el cáncer de piel ocupó el primer lugar en incidencia entre las mujeres y ocupó el segundo lugar en incidencia entre los hombres entre 1994 y 1996 (Bartels, Vargas, y Muñoz, 1999). Aunque su tasa de mortalidad fue muy baja ( 0,6 y 0,7 por cada 100000 habitantes, en mujeres y hombres, respectivamente), su incidencia empieza a ser importante a partir de los 40 años de edad. Es más, según datos del Registro Nacional de Tumores del Ministerio de Salud de Costa Rica, el 65\% de los tumores de piel se presentaron en mujeres y hombres de 65 años y mayores (Bartels, et. al, 1999). Aunque las posibles causas de estas elevadas tasas de incidencia aún no se han determinado, se podría especular que una buena parte de la varianza podría explicarse por la exposición prolongada y crónica a los rayos solares, la cual a su vez, estaría relacionada con ocupaciones o actividades en las que las personas están expuestas durante 
más tiempo al sol (e.g., agricultores que trabajan en zonas donde la mayor parte del año hay una fuerte radiación solar, educadores físicos y atletas que entrenan a horas en las que hay mayor radiación solar).

Ya que existen diversos tipos de cáncer de piel, siendo los melanomas los de mayor mortalidad, es imperativa la detección temprana de irregularidades en la piel, especialmente en aquellas partes expuestas constante y crónicamente al sol, como por ejemplo, los brazos, el cuello, el rostro, las orejas y el escote (Franceschi, Levi, Randimbison, y La Vecchia, 1996). La revisión periódica de estas zonas anatómicas es especialmente importante en aquellos educadores físicos que imparten clases de natación, o que no acostumbran a utilizar prendas que les brinde protección contra los rayos UV, como por ejemplo, camisetas de manga larga, pantalón o buzo deportivo, gorra o sombrero, anteojos oscuros, y lociones bloqueadoras con un factor de protección solar (SPF por sus siglas en inglés) indicado por el dermatólogo.
Si se detectan y controlan a tiempo, estos tipos de cáncer pueden curarse (Muglia, Pesce, y McDonald, 1999). De acuerdo con Tortora y Grabowski (1993), todas las personas en riesgo deben autoexaminarse periódicamente, tratando de encontrar lunares que aumentan de tamaño, que desarrollen bordes muy irregulares, superficies desiguales, o una mezcla de colores. Si el lunar o zona afectada es detectada a tiempo, se puede recurrir a una extirpación quirúrgica, especialmente cuando el cáncer es basocelular o epidermoide (Tortora y Grabowski, 1993). Por ello, se recomienda seguir la regla del "abecedario" para el examen personal de la piel (Jerant et al., 2000) (Tabla 1).

En el autoexamen, se debe poner especial atención a un lunar que sangra, un lunar que crece rápido, un lunar que no sana, un lunar que produce comezón, y una parte de la piel que se siente áspero, como papel de lija. Finalmente, se recomienda visitar al médico dermatólogo si un lunar cambia de alguna manera en su forma, color, tamaño o textura (Jerant et al., 2000).

Tabla 1

La Regla del “ABCDEF” para el autoexamen de la piel

\begin{tabular}{ll}
\hline Asimetría & $\begin{array}{l}\text { Cuando los dos lados del lunar no lucen iguales se puede estar ante la presencia de le- } \\
\text { siones de tipo melanoma. }\end{array}$ \\
Bordes & $\begin{array}{l}\text { El cáncer de tipo melanoma generalmente presenta lesiones en las cuales los bordes del } \\
\text { lunar son borrosos, dentados, o escamosos. }\end{array}$ \\
Color & $\begin{array}{l}\text { El color del lunar cambia -si es más oscuro que antes, el color se expande o se pierde, o } \\
\text { aparece más de un color (azul, rojo, blanco, rosado, morado, marrón, o gris). }\end{array}$ \\
Diámetro & $\begin{array}{l}\text { Cuando el diámetro del lunar es mayor a } 6 \text { mm (más o menos del tamaño del borrador } \\
\text { de un lápiz). }\end{array}$ \\
Elevación & Cuando el lunar sobresale de la piel y presenta un aspecto áspero en su superficie. \\
Familia & $\begin{array}{l}\text { Tomar precauciones extra cuando le han extirpado un tumor de piel anteriormente, o si } \\
\text { tiene antecedentes familiares de cáncer de piel. }\end{array}$ \\
\hline
\end{tabular}

Modificado de: Jerant, A. F., Johnson, J. T., Sheridan, C. D., \& Caffrey, T. J. "Early detection and treatment of skin cancer". American Family Physician. 62, 357-368, 375-376, 381-382. 2000. 
Sería recomendable transmitir a los estudiantes este mensaje de forma oral y escrita, con el propósito de que los padres y encargados puedan tomar las previsiones del caso y que aprendan a autoexaminarse en la privacidad de su hogar.

Otra recomendación principal se refiere a la aplicación de cremas protectoras o "bloqueadoras" (no son lo mismo que "bronceadoras") con un SPF por lo menos de 15, prendas protectoras como gorras o sombreros, lentes oscuros, y la programación de entrenamientos y competencias en horas de baja radiación UV. Se sabe que las horas de mayor radiación UV son entre 10:00 a.m. y 4:00 p.m. (Jerant et al., 2000; Moehrle et al., 2000), por lo que idealmente las clases de educación física deberían programarse fuera de ese horario; pero como no es factible acatar esa recomendación, se debería programar las clases de los niños más pequeños muy temprano en la mañana (i.e., 7:00 a 9:00 a.m.) y dejar que los niños de mayor edad realicen las actividades después de las 10:00 a.m. asegurándose de que utilicen una adecuada protección contra los rayos UV.

También se deben realizar campañas educativas en las que se incluya la evaluación de grupos en alto riesgo, como por ejemplo, hombres y mujeres blancas, rubias, pelirrojas, de ojos color claro, atletas que entrenan en el día, surfeadores y otros deportistas acuáticos, ciclistas, triatlonistas, futbolistas, niños, adultos mayores, y en general, aquellos que entrenan o compiten durante horas con la mayor radiación solar (Gentile y Auerbach, 1987; Dozier, Wagner, Black, y Terracina, 1997).

Tortora y Grabowski (1993), mencionan que a pesar de que en algunos establecimientos en los que se promociona un bronceado artificial se afirma que las longitudes de onda de los rayos UV no son dañinas, se ha encontrado que tanto las longitudes de onda UV amplia (UVA) y corta (UVB) son igualmente peligrosas, y que además pueden acarrear otros daños adicionales para el organismo, como por ejemplo, envejecimiento prematuro de la piel, inhibición del sistema inmunológico, y lesiones en los ojos a nivel del cristalino y de la retina. Es más, en Suiza se ha encontrado que aún exposiciones de corta duración a los rayos UV, son capaces de aumentar el riesgo de adquirir un melanoma cutáneo maligno (Franceschi, Levi, Randimbison, y La Vecchia, 1996; Rosso et al., 1996).

Futuras investigaciones longitudinales deberían tomar en cuenta factores como el historial médico, historia de reproducción, tipo de entrenamiento, duración, intensidad, dieta, y otros hábitos, los cuales, desde un punto de vista metodológico, son variables confusoras importantes que podrían brindar mayor información acerca de los posibles riesgos asociados a la exposición a los rayos UV. En Costa Rica, se deben empezar a realizar investigaciones en educadores físicos y obtener estadísticas de este grupo de trabajadores que sirvan para diseñar guías específicas.

Finalmente, no se debe privar a las personas de realizar actividades físicas en días soleados, ya que la activación de la vitamina $\mathrm{D}$ podría verse comprometida. Si una persona no recibe suficiente radiación solar, ésta podría comprometer la absorción del calcio y el fósforo en el tracto gastrointestinal, lo cual, en condiciones extremas, podría causar raquitismo en los niños y osteomalacia en los adultos, además de una posible pérdida del tono muscular (Tortora y Grabowski, 1993). Por ello, una adecuada campaña educativa es la mejor herramienta para disfrutar inteligentemente de los beneficios del sol.

\section{Referencias bibliográficas}

Bartels, R., Vargas, R. M., \& Muñoz, G. Incidencia y mortalidad del cáncer en Costa Rica: 1994 - 1996. San José, Costa Rica: Ministerio de Salud. 1999. 
Bernstein, L., Henderson, B. E., Hanisch, R., Sullivan-Halley, J., \& Ross, R. K. "Physical exercise and reduced risk of breast cancer in young women". Journal of the National Cancer Institute. 86(18), 1403-1408. 1994.

Cohen, P. R., Eliezri, Y. D., \& Silvers, D. N. "Athlete's nodules: sports-related connective tissue nevi of the collagen type (collagenomas)". Cutis. 50(2), 131-135. 1992.

Coogan, P. F., Newcomb, P. A., Clapp, R. W., Trentham-Dietz, A., Baron, J. A., \& Longnecker, M. P. "Physical activity in usual occupation and risk of breast cancer (United States)". Cancer Causes Control. 8(4), 626-631. 1997.

Creagh, T. M., Rubin, A., \& Evans, D. J. "Hepatic tumours induced by anabolic steroids in an athlete". Journal of Clinical Pathology. 41(4), 441-443. 1988.

Dozier, S., Wagner, R. F. Jr., Black, S. A., \& Terracina, J. "Beachfront screening for skin cancer in Texas Gulf coast surfers". Southern Medical Journal. 90(1), 55-58. 1997.

Ford, E. S. "Body mass index and colon cancer in a national sample of adult US men and women". American Journal of Epidemiology. 150(4), 390-398. 1999.

Franceschi, S., Levi, F., Randimbison, L., \& La Vecchia, C. "Site distribution of different types of skin cancer: new aetiological clues". International Journal of Cancer. 3;67(1), 24-28. 1996.

Friedenreich, C. M., \& Rohan, T. E. "Physical activity and risk of breast cancer". European Journal of Cancer Prevention. 4(2), 145-151. 1995.
Frisch, R. E., Wyshak, G., Albright, N. L., Albright, T. E., Schiff, I., Jones, K. P., Witschi, J., Shiang, E., Koff, E., \& Marguglio, M. "Lower prevalence of breast cancer and cancers of the reproductive system among former college athletes compared to non-athletes". British Journal of Cancer. 52(6), 885-891. 1985.

Frisch, R. E., Wyshak, G., Witschi, J., Albright, N. L., Albright, T. E., \& Schiff, I. "Lower lifetime occurrence of breast cancer and cancers of the reproductive system among former college athletes". International Journal of Fertility. 32(3), 217-225. 1987.

Frisch, R. E., Wyshak, G., Albright, N. L., Albright, T. E., \& Schiff, I. "Lower prevalence of non-reproductive system cancers among female former college athletes". Medicine and Science in Sports and Exercise. 21(3), 250-253. 1989.

Gentile, D. A., \& Auerbach, P. S. "The sun and water sports". Clinics in Sports Medicine. 6(3), 669-684. 1987.

Jerant, A. F., Johnson, J. T., Sheridan, C. D., \& Caffrey, T. J. "Early detection and treatment of skin cancer". American Family Physician. 62, 357-368, 375-376, 381-382. 2000.

Mayerhausen, W., \& Riebel, B. "Acne fulminans following use of anabolic steroids". Z Hautkr. 15;64(10), 875-876, 879-880. 1989.

Moehrle, M., Heinrich, L., Schmid, A., \& Garbe, C. "Extreme UV exposure of professional cyclists". Dermatology. 201(1), 44-45. 2000.

Mottram, D. R., \& George, A. J. "Anabolic steroids". Baillieres Best Practice 
Research on Clinical Endocrinology Metabolism. 14(1), 55-69. 2000.

Muglia, J. J., Pesce, K., \& McDonald, C. J. "Skin cancer screening: A growing need". Surgical Oncology Clinics of North America. 8(4), 735-745. 1999.

Pukkala, E., Kaprio, J., Koskenvuo, M., Kujala, U., \& Sarna, S. "Cancer incidence among Finnish world class male athletes". International Journal of Sports Medicine. 21(3), 216220. 2000.

Rockhill, B., Willett, W. C., Hunter, D. J., Manson, J. E., Hankinson, S. E., \& Colditz, G. A. "A prospective study of recreational physical activity and breast cancer risk". Archives of Internal Medicine. 159(19), 2290-2296. 1999.

Rosso, S., Zanetti, R., Martinez, C., Tormo, M. J., Schraub, S., Sancho-Garnier, H., Franceschi, S., Gafa, L., Perea, E., Navarro, C., Laurent, R., Schrameck, C., Talamini, R., Tumino, R., \& Wechsler, J. "The multicentre south European study "Helios" II: Different sun exposure patterns in the aetiology of basal cell and squamous cell carcinomas of the skin". British Journal of Cancer. 73(11), 1447-1454. 1996.

Rosso, S., Joris, F., \& Zanetti, R. "Risk of basal and squamous cell carcinomas of the skin in Sion, Switzerland: a case-control study". Tumori. 85(6), 435-442. 1999.

Tortora, G. J., \& Grabowski, S. R. Principios de anatomía y fisiología (2da reimpresión). España: Harcourt Brace de España, S.A. 1993.

Thune, I., Brenn, T., Lund, E., \& Gaard, M. "Physical activity and the risk of breast cancer". New England Journal of Medicine. 336(18), 1269-1275. 1997.

van Dam, R. M., Huang, Z., Rimm, E. B., Weinstock, M. A., Spiegelman, D., Colditz, G. A., Willett, W. C., \& Giovannucci, E. "Risk factors for basal cell carcinoma of the skin in men: results from the health professionals follow-up study". American Journal of Epidemiology. 150(5), 459-468. 1999.

Verloop, J., Rookus, M. A., van der Kooy, K., \& van Leeuwen, F. E. "Physical activity and breast cancer risk in women aged 20-54 years". Journal of the National Cancer Institute. 92(2), 128-135. 2000.

Wyshak, G., \& Frisch, R. E. "Breast cancer among former college athletes compared to non-athletes: a 15-year follow-up". British Journal of Cancer. 82(3), 726-730. 2000.

José Moncada Jiménez

Profesor de la Escuela de

Educación Física y Deportes

Universidad de Costa Rica 